\title{
Counterstreaming beams in magnetised Vlasov plasma
}

\author{
L PALODHI $^{1}{ }^{10}, *$, F CALIFANO $^{2}, \mathrm{M} \mathrm{E} \mathrm{DIECKMANN}^{3}$ and $\mathrm{F} \mathrm{PEGORARO}^{2}$ \\ ${ }^{1}$ Department of Mathematics, Iowa State University, Ames, IA, USA \\ ${ }^{2}$ Department of Physics, University of Pisa, Pisa, Italy \\ ${ }^{3}$ Department of Science and Technology (ITN), Linkoping University, Norrköping, Sweden \\ *Corresponding author. E-mail: lpalodhi@iastate.edu
}

MS received 31 May 2018; revised 27 June 2019; accepted 18 July 2019

\begin{abstract}
In this paper, we investigate nonrelativistic, kinetic, linear phase of the filamentation instability when an external magnetic field is present in the direction of the counterstreaming electron beams using Vlasov simulations in 1D-3V space. We first investigate the growth rate of instability. In the linear growth regime, our results correspond to the previous conclusions that with the increase in strength of the ambient magnetic field, there is a suppression of instability. Interestingly, we established that at a critical/threshold magnetic field, Vlasov simulations and particlein-cell (PIC) simulations differ in their instability behaviour. At this particular magnetic field, there is a complete suppression of the growth of instability in Vlasov results compared to PIC simulations, where a strong growth of instability is shown. It is believed that thermal noise in the PIC leads to the growth. However, Vlasov simulations show wave-wave coupling which stabilises the modes. In this work, our focus is to demonstrate the difference in this behaviour and to thoroughly analyse the spectra and wave generation for the same.
\end{abstract}

Keywords. Counterstreaming beams; magnetised plasma; marginal instability.

PACS Nos 52.65.Ff; 52.25.Xz; 52.65. $-\mathrm{y} ;$ 52.35. $-\mathrm{g}$

\section{Introduction}

The Weibel instability [1], named after E S Weibel who first showed analytically the growth of instability in an unmagnetised plasma with a temperature anisotropy, is often referred to as the filamentation instability (FI) or the beam-Weibel instability when counterstreaming beams play the role of anisotropy. The filamentation instability has been proposed as a mechanism to magnetise the early Universe [2], to provide strong magnetic fields for the afterglow emission of $\gamma$-ray bursts [3] and supernovae explosions [4], and to explain the heating processes in the pulsar winds [5]. The FI has also been investigated to explain the generation of quasistatic magnetic fields in the interaction of ultrashort and ultraintense laser pulses with an underdense plasma [6-8]. FI is present in laser fusion plasmas. Stabilisation effects due to the coupling of the self-generated magnetic field by Weibel instability with the laser wave field has also been shown explicitly [9]. Two-stream instability generated from FI is shown to be present in laser Wakefield-driven plasma waves as well [10]. In very different conditions, it has been reconsidered under the name of 'Chromo-Weibel instability' in relativistic heavy-ion collision experiments, having been reformulated within a quantum chromodynamic framework [11].

The free energy stored in the counterstreaming electrons in the presence of static background ions is released by the well-known two-stream electrostatic instability and by the transverse electromagnetic FI. The two-stream instability in an unmagnetised plasma is completely electrostatic while it has an electromagnetic nature in magnetised plasmas when the stream velocities become relativistic [12]. The physical mechanism that drives the electromagnetic perturbations can be understood by remembering that, when the electric currents carried by the two electron streams are displaced, one with respect to the other, by a transversal disturbance, the repulsion of the two oppositely directed currents reinforces the initial displacement. As a result, the magnetic field grows in time.

The FI has been thoroughly investigated analytically and numerically within the fluid $[13,14]$ model. The literature on numerical investigations of the FI in the kinetic regime is also vast. The FI has been 
investigated with a one-dimensional (1D) Vlasov code $[15,16]$ and with 1D [17,18] and two-dimensional [19] particle-in-cell (PIC) codes. It has also been studied with 3D PIC simulations [20]. Laboratory experiments have also been made to study the counterstreaming instability [21].

In the present paper, we extend the previous work on FI and investigate the system in detail when a background external magnetic field is present. The nonrelativistic, kinetic, linear phase of the instability is analysed. Vlasov simulations in $1 \mathrm{D}-3 \mathrm{~V}$ phase space are used where only the electrons are considered to be in motion while the ions are fixed at the background.

We assume two opposite streams of electrons. We consider identical absolute velocities for both streams, and equal densities, because it was shown in earlier studies [14] that the growth rate of the FI, relative to that of the two-stream and oblique modes, is largest if both beams have equal density, composition and temperature. In the absence of a static electric field perpendicular to the magnetic field lines, the background magnetic field is assumed to be parallel to the streaming direction. In this constrained 1D configuration, we assume periodic boundary conditions along the direction of the FI wave number. The electric field generated by the FI is in the direction perpendicular to the direction of FI while the two-stream instability would generate an electric field in the same direction as FI. In reality, the FI competes with the two-stream instability and it cannot be observed in an isolated form, even if the equal beam density case favours the FI over the two-stream instability [22]. However, in our simple 1D configuration, as the spatial dependence is only along one direction, i.e. the direction of streaming beams, we cannot observe the two-stream instability. Thus, the dynamics of our system becomes relatively simpler as we only see the FI. An electrostatic field is generated but it is not due to the two-stream instability but due to the electromagnetic perturbations themselves, as described in ref. [23].

The present paper consists of two main sections. The first part looks into the growth rate of instability, and how the magnitude of the ambient magnetic field affect the FI. In this part of the work, we show that when an initial magnetic field is applied along the streaming direction, the FI develops, and the strength of the magnetic field generated by the instability decreases as the magnitude of the ambient magnetic field increases. Above a critical magnetic field, the FI is no longer excited. In earlier works [24] it was suggested that at the critical magnetic field there is a growth of instability due to 'simulation noise'. From our Vlasov simulation runs, where thermal noise is absent, we first verify that the temperature effects stabilise the FI. Then, in order to determine whether an initial strong noise characteristic of PIC simulations could be the cause of the threshold effect described in ref. [24], we performed a number of Vlasov numerical runs adding different levels of wave noise in the form of density and magnetic field perturbations at the initial time. However, no difference in our simulation results were observed as we varied the initial amplitude of the perturbations. Thus, we interpret that the presence of an initial noise per se is not sufficient to explain the mode growth at the threshold found in ref. [24]. Hence, we argue that the PIC simulations and Vlasov simulations give different results because PIC codes have thermal noise due to the random motion of the particles compared to the wave noise due to random waves in Vlasov code. Our arguments have been discussed and confirmed by studying all the different kinds of waves generated during the process and also with various detailed spectral analysis of the waves in the linear regime.

This paper is structured as follows: Governing equations and numerical model of the system are discussed in $\S 2$ which is followed by the numerical results in $\S 3$. Section 4 concentrates on some of preliminary results on the saturation phase of instability and finally conclusions are drawn in $\$ 5$.

\section{Simulation model}

A homogeneous plasma equilibrium configuration is considered with two counterpropagating symmetric (identical) beams moving along the $y$ direction. Collisions are neglected and the plasma evolution is described by the Vlasov-Maxwell system of equations taking the ions to be a fixed at the background. The integration of the Vlasov-Maxwell equations is performed in the $\left(x, v_{x}, v_{y}, v_{z}\right)$ phase space of electrons with the following initial conditions:

$f_{0}(x, v, 0)=f_{\mathrm{M}}\left(v_{x}, v_{y}, v_{z}\right)\left[1+\left(\sum_{n=1}^{N} c_{i} \sin \left(k_{n} x+\chi_{n}\right)\right)\right]$
$k_{n}=2 \pi n / L_{x}$

where $f_{\mathrm{M}}$ is the initial (non-relativistic) bi-Maxwellian given by

$$
\begin{aligned}
f_{\mathrm{M}}\left(v_{\mathrm{x}}, v_{y}, v_{z}\right)= & \frac{n_{e}}{2 \pi^{3 / 2} v_{\text {the }}^{3}} \mathrm{e}^{-\left(v_{x}^{2}+v_{z}^{2}\right) / v_{\text {the }}^{2}}\left[\mathrm{e}^{-\left(v_{y}-v_{0}\right)^{2} / v_{\text {the }}^{2}}\right. \\
& \left.+\mathrm{e}^{-\left(v_{y}+v_{0}\right)^{2} / v_{\text {the }}^{2}}\right],
\end{aligned}
$$

where $v_{\text {the }}$ is the thermal velocity and $v_{0}$ is the velocity of the electron streams. We take $v_{0} / c=0.2>v_{\text {the }} / c$, where $c$ is the speed of light. We also apply an initial magnetic noise in the form of randomly oriented magnetic fields: 


$$
\begin{aligned}
& B_{y}(x, t=0)=\sum_{n=1}^{N} a_{i}\left[\cos \left(k_{n} x+\psi_{n}\right)\right], \\
& B_{z}(x, t=0)=\sum_{n=1}^{N} b_{i}\left[\cos \left(k_{n} x+\phi_{n}\right)\right]
\end{aligned}
$$

with small perturbed initial amplitudes $a_{i}, b_{i}$ and $c_{i}$ and $\psi_{n}, \phi_{n}$ and $\chi_{n}$ are random phases. The initial electric field and the electric current are set to zero.

\section{Numerical results}

\subsection{Linear evolution of instability}

The numerical parameters used are as follows: $x$ direction has $N_{x}=64$ grid points and the total length of the simulation box is $L_{x}=10 \pi d_{e}$, where $d_{e}=c / \omega_{p e}$ is the electron collisionless skin depth, so that the grid spacing is $d x=0.49 d_{e}$ and the lowest wave number is $d k=2 \pi / L_{x}$. The number of grid points in velocity space are $N v_{x}=N v_{z}=25$ and $N v_{y}=100$. The number of modes is $N=30$. Although, the number of grid points is low and number of modes chosen is less than what should be available, the lower number of available modes do not change our study on the critical magnetic field significantly. We let the system evolve up to $t=250 \omega_{p e}^{-1}$. The initial amplitude of the fields is set at $a_{i}=b_{i}=c_{i}=10^{-2}$. In the following we use normalised quantities. We use the plasma frequency $\omega_{p e}$ and the velocity of light $c$ as characteristic frequency and velocity. Therefore, the electron skin depth $c / \omega_{p e}$ is the characteristic length scale. The electric and magnetic fields are normalised to $\bar{E}=c \bar{B}=m_{e} c \omega_{p e} / e$. The wave number $(k)$ is normalised to the electron skin depth $\left(c / \omega_{p e}\right)$.

Table 1 shows the linear growth rate obtained by our numerical simulations for different strengths of ambient magnetic fields imposed to the system with thermal velocities, $v_{\text {the }} / c=0.03$ (which is approximately equal to the cold fluid limit) and $v_{\text {the }} / c=0.1$. The table also includes the analytical values of growth. The analytical growth rate for kinetic and fluid limits is calculated using the equation given in [24].

$$
\begin{aligned}
\gamma_{\mathrm{ana}}= & \left\{-\frac{1}{2}\left(\omega_{c e}^{2}+\omega_{p e}^{2}+k^{2} c^{2}\right)+\left[\frac{\left(\omega_{c e}^{2}+\omega_{p e}^{2}+k^{2} c^{2}\right)^{2}}{4}\right.\right. \\
& \left.\left.-k^{2} c^{2} \omega_{c e}^{2}-\omega_{p e}^{2} \omega_{c e}^{2}+\omega_{p e}^{2} k^{2}\left(v_{0}^{2}+\frac{v_{\text {the }}^{2}}{2}\right)\right]^{1 / 2}\right\}^{1 / 2} .
\end{aligned}
$$

The values of the numerical and analytical growth rates differ maximum by $10 \%$ and that too in the fluid limit,
Table 1. A comparison of the numerical growth rates with $N_{x}=64$.

\begin{tabular}{llllcc}
\hline$B_{0}$ & $v_{\text {the }}$ & $k_{\max }$ & $\gamma_{\max }^{\mathrm{Num}}$ & $\gamma_{\text {kin }}^{\mathrm{Ana}}$ & $\gamma_{\text {flu }}^{\mathrm{Ana}}$ \\
\hline 0.0 & 0.03 & 1.2 & 0.25 & 0.154 & 0.153 \\
& 0.1 & 1.0 & 0.15 & 0.150 & 0.140 \\
0.1 & 0.03 & 1.2 & 0.20 & 0.117 & 0.116 \\
& 0.1 & 1.0 & 0.13 & 0.111 & 0.019 \\
0.15 & 0.03 & 1.2 & 0.075 & 0.037 & 0.033 \\
& 0.1 & 1.0 & 0.045 & - & - \\
\hline
\end{tabular}

$v_{\text {the }}=0.03$. This difference is reduced to $4 \%$ as we move towards stronger ambient magnetic fields. The difference is only $2 \%$ in the kinetic limit with $v_{\text {the }}=0.1$. Growth rates decrease as the magnetic field is increased from $B_{0}=0$ to 0.15 . The growth rate at $B_{0}=0$ is almost thrice as that of the growth of instability at $B_{0}=0.15$. However, the wave mode at which the maximum growth rate is observed is found to be at $k_{\max }=1$ for all cases with thermal velocity $v_{\text {the }}=0.1$, independent of $B_{0}$. We also see that when we decrease the thermal velocity from $v_{\text {the }}=0.1$ to 0.03 , the numerical growth rate increases by a factor of almost two, and the wave mode corresponding to the maximum growth rate increases, thereby showing the stabilising effect of the beam's temperature. In figure 1 the numerical dispersion relation for $B_{0}=0.0$ and 0.1 is plotted and we found that the behaviour of the system changes when it goes from unmagnetised to magnetised system. In the top panel we see that at low $k$ region, $0<k<0.2$, the growth of the wave is actually a little higher when the system is under strong thermal effect, but then for high $k$ values the growth is always stronger for lower thermal velocity. However, in magnetised system, even at low $k$ values, the growth is higher for $v_{\text {the }}=0.03$ and hence we can observe a stabilisation of temperature. The physical reason for the quenching of the FI at high wave numbers is the thermal motion of the particles. The repulsion of counterstreaming particles tries to create the filaments, while their thermal motion tries to counteract this rearrangement. At large $k$, the thermal effects are the strongest. It is difficult for filaments to form. However, thermal effects are small for long wavelengths and hence growth rate is strong for the FIs.

The growth of the fastest waves has also been studied using grid point $N_{x}=128$, with number of modes $N=60$. Comparison of the growth rates is shown in table 2. The growth rates are better approximated with higher resolution. The difference in the numerical and analytical rates is just around $1 \%$ in the absence of ambient magnetic field. However, this difference is approximately $5 \%$ as we advance to higher magnetic field and also at the critical magnetic field. Using larger 

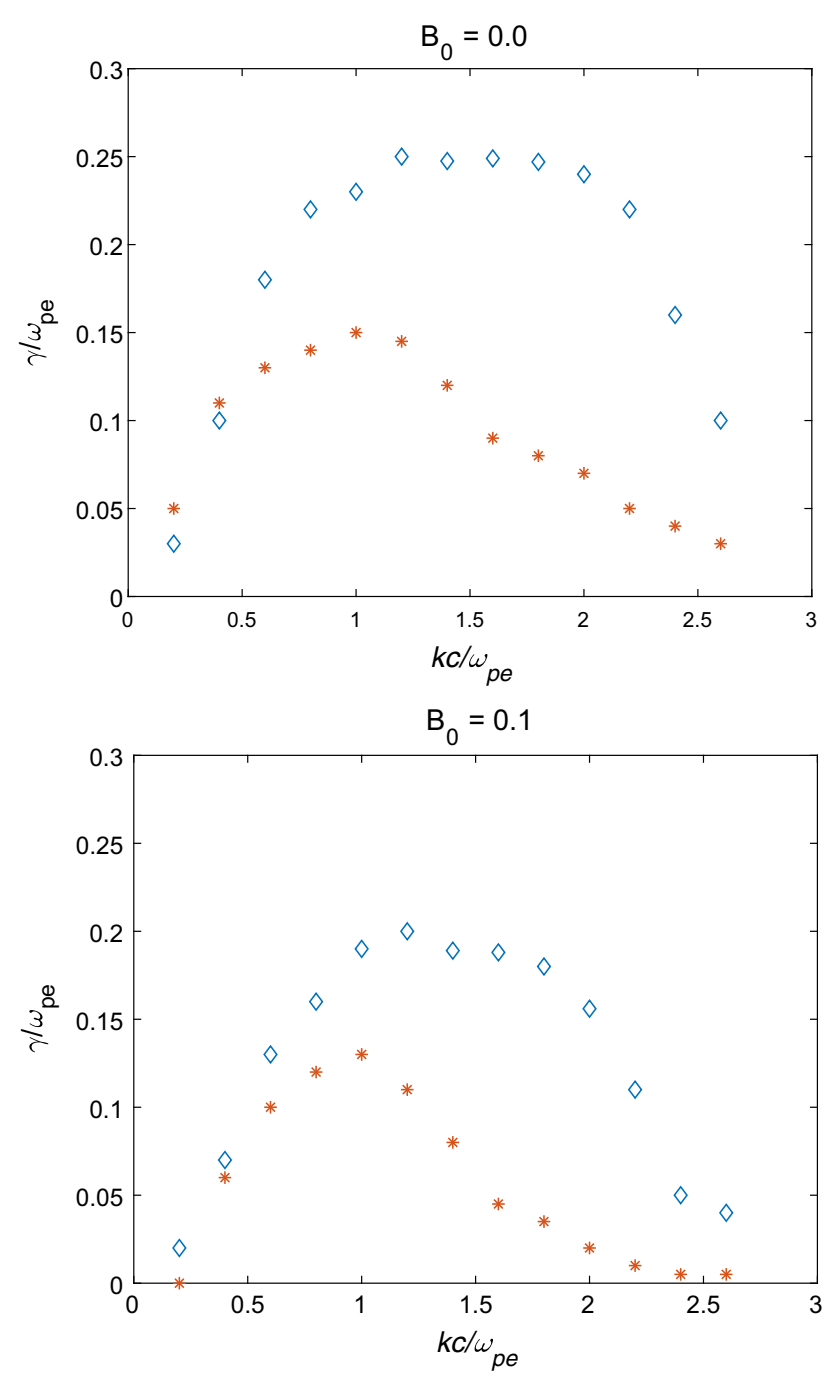

Figure 1. Top panel: Numerical Vlasov growth rate for unmagnetised plasma, where diamonds stand for $v_{\text {the }}=0.03$ and stars stand for $v_{\text {the }}=0.1$. Bottom panel: Numerical Vlasov growth rate for magnetised plasma $\left(B_{0}=0.1\right)$.

Table 2. Numerical growth rates with $N_{x}=128$.

\begin{tabular}{llll}
\hline$B_{0}$ & $v_{\text {the }}$ & $k_{\max }$ & $\gamma_{\max }^{\mathrm{Num}}$ \\
\hline 0.0 & 0.03 & 1.2 & 0.150 \\
& 0.1 & 0.8 & 0.085 \\
0.1 & 0.03 & 1.2 & 0.130 \\
& 0.1 & 0.8 & 0.064 \\
0.15 & 0.03 & 1.2 & 0.085 \\
& 0.1 & 0.8 & 0.040 \\
\hline
\end{tabular}

number of grid points results in a progressively smaller difference between the analytical growth rates. Qualitatively, however, the results and trends remain the same. Our goal, in this case, is to demonstrate that the growth of instability is suppressed as the background magnetic field is increased, and specifically, there is no growth of instability at the critical magnetic field.

\subsection{Stable modes at the critical magnetic field}

As we increase the strength of the magnetic field, the FI is suppressed and above a critical magnetic field the growth of the wave is inhibited and the system becomes stable. This is confirmed using our Vlasov simulation with the magnetic field $B_{0}=0.5$. In this case, $\omega_{c e}>\omega_{p e} v_{0} / c$. Hence, we have a critical magnetic field $B_{\mathrm{c}}$ above which the instability vanishes. In dimensional units, $B_{\mathrm{c}}$ is expressed as

$B_{\mathrm{c}}=\left(4 \pi n m_{e}\right)^{1 / 2} v_{0}$

which corresponds to the magnetic field energy density becoming equal to the beams's kinetic energy density. The FI is stable for $B_{0}>B_{\mathrm{c}}$ because the electrons are magnetically confined and cannot redistribute themselves in space, which is required for the development of the FI. Quantitatively, this stabilisation can be traced back to the fact that in the presence of an ambient magnetic field, the conservation of the beams's canonical momentum along $z$ requires that in order to displace the beams along $x$, energy should be transferred to the particle motion along $z$.

In a real plasma, at marginal stability, a large increase in the electron density fluctuations can be expected from the thermal noise [25] due to the "phase-space granularity'. For example, in the context of the study of ballooning stability in an igniting plasma, it was shown [26] that magnetic fluctuations driven by the fusion reaction products and capable of achieving significant amplitudes can be excited in a plasma regime close to the so-called 'second stability' region. Similar type of fluctuations has apparently also been observed in the 1D PIC simulations of the FI in a magnetised plasma reported in ref. [24] where an unexpected growth of magnetic fluctuations at the critical magnetic field $B_{0}=B_{\mathrm{c}}$ was found. Hence, it is interesting to see whether this effect can be reproduced in a Vlasov code where the meanfield Maxwell equations are integrated as in PIC codes but the numerical noise, due to the finite number of orbits sampled in PIC codes, is absent.

From ref. [24] we found that the threshold magnetic field is obtained when $\omega_{c e}=\left(\omega_{p e} / c\right)\left(v_{0}^{2}+v_{\text {the }}^{2} / 2\right)^{1 / 2}$. So, when thermal effects are neglected $\left(v_{0} \gg v_{\text {the }}\right)$, we arrive at the fluid limit of the threshold magnetic field, i.e., $\omega_{c e}=\omega_{p e} v_{0} / c$. As $v_{\text {the }}$ is increased, threshold magnetic field will rather depend on $v_{\text {the }}$ instead of $v_{0}$. For $v_{\text {the }}=0.03$ and $v_{0}=0.2$, the cold limit threshold is $B_{\mathrm{c}}=0.2$ and including thermal effects, the critical magnetic field is at $B_{\mathrm{c}}=0.201$. Hence we consider both to be approximately the same. We take the ambient 
Table 3. Simulation runs which show that instability stabilises at the threshold and no marginal instability exists.

\begin{tabular}{lccc}
\hline$B_{0}$ & $v_{\text {the }}$ & $a_{i}=b_{i}=c_{i}$ & $\gamma_{\max }^{\text {Num }}$ \\
\hline 0.18 & 0.03 & $10^{-2}$ & 0.020 \\
0.19 & 0.03 & $10^{-2}$ & 0.019 \\
0.2 & 0.03 & $10^{-2}$ & - \\
0.21 & 0.03 & $10^{-2}$ & - \\
0.22 & 0.03 & $10^{-2}$ & - \\
\hline
\end{tabular}

magnetic field at its threshold value, $B_{0}=0.2$. In the first set of simulation runs we consider the initial wave noise of the magnetic field and the electron density to be of order $10^{-4}$ and consider both low and high thermal velocities, $v_{\text {the }}=0.03$ and 0.1 respectively. However, no amplification of the fluctuations is observed even when the initial noise of the density and magnetic field fluctuations is increased up to order $10^{-1}$. In order to cross check that the critical magnetic field is exactly at $\omega_{c e}=\omega_{p e} v_{0} / c$ (according to fluid calculations), we made some additional simulation runs by increasing and decreasing the ambient magnetic field by 5 and $10 \%$, as shown in table 3 . At $B_{0}=0.18$ and 0.19 we still observe the growth of magnetic field regardless of the fact that the growth rate is reduced. Above the threshold value, we see no such growth even in the presence of a strong initial noise in both the electron density and in the magnetic field. To show that marginal instability is indeed due to the numerical noise in PIC codes, we carried out some additional simulations adding random fluctuations in the Vlasov code. We also tried by adding external electrostatic forces at different times at a threshold magnetic field with $B_{0}=v_{0}=0.2$. However, in this case too we did not observe any growth of instability. This is suggestive of a different mechanism for the marginal instability observed by PIC codes. The noise, which is not present in Vlasov codes, is caused by the granular structure of the phase space density distribution in PIC simulations. Some recent studies throw light in this direction. Recently, it has been observed that gyrokinetic simulations of electron temperature gradient (ETG) turbulence with PIC code yielded different results from flux-tube continuum code simulations, despite similar parameters. This difference in their results was attributed to insufficient phase-space resolution and was shown to result from discrete particle noise [27], which is a numerical artifact. A convergence study has also been done in this regard. Similar work has been also shown by Bottino et al [28], and have shown that global, nonlinear, PIC simulations of electron temperature-driven turbulence recover the same level of transport as flux-tube codes when the level of the statistical noise, associated with the PIC discretisation is sufficiently small. Hence, we believe PIC codes will result in similar numerical artifact for any kinetic instabilities. In general, particle noise present in PIC simulation yields results that differ from continuum models.

Consider a computational particle. It has a localised charge and current density distribution and it moves around and drives waves. Their phase speed thus equals the particle speed. Most electrons are slower than the phase velocity of the Langmuir wave and the waves driven by the electron motion are thus damped modes because they are too slow to get onto the dispersion relation of the Langmuir mode. These damped waves thus disappear rapidly as we go away from the particle (strong imaginary $k$ ). So each computational particle drags with it a small electric and magnetic field pulse. Other particles, which are moving across this particle, will get a small kick by the wave fields. If a particle collides with many of these, it gets a series of random kicks. This type of wave-particle collision shows up, because PIC codes solve the Klimontovich equation and not the Vlasov equation. This is illustrated in ref. [29]. Further, a Vlasov code cannot model the thermal noise, which reflects the random motion of individual particles. In Vlasov model, we place random waves in the system, which will then propagate as linearly undamped Bernstein mode waves and upper hybrid waves. So Vlasov model has wave noise rather than thermal noise, which causes marginal instability in PIC codes. This is further verified using spectral analysis in the following subsection.

\subsection{Spectra and wave generations}

The magnitudes of the background magnetic field were chosen to be $B_{0}=0.1$ and 0.5 , for the spectral study, such that the ratio of plasma frequency and the cyclotron frequency is of the order of 10 and 2 respectively. The thermal velocity considered is $v_{\text {the }}=0.1 . x$ direction has $N_{x}=1024$ grid points and total length of the box selected is $L_{x}=100 \pi d_{\mathrm{e}}$, so that grid spacing $d x=$ $0.03 d_{e}$ and the lowest wave number $d k=2 \pi / L_{x}$. The number of grid points in the velocity space are $N v_{x}=$ $N v_{z}=30$ and $N v_{y}=50$. The number of modes $N=$ 250. Let the system evolve up to $t=450 \omega_{\mathrm{pe}}^{-1}$. The initial amplitude of the fields is set at $a_{i}=b_{i}=c_{i}=10^{-4}$.

Contour plots of electric fields for $B_{0}=0.1$ in $\left(\omega_{r}-k\right)$ plane are shown in figure 2 . The electric field $E_{y}$ is the inductive field of the FI. The beams flow along $y$, the magnetic field grows along $z$ and the only part of the rotational $B$ is the $E_{y}$-component. This one couples to $E_{y}$ of the O-mode. It grows proportionally to rotational $B$ and, thus, to $B$. The electric field $E_{y}$ can otherwise only grow along $x$ during the non-linear 


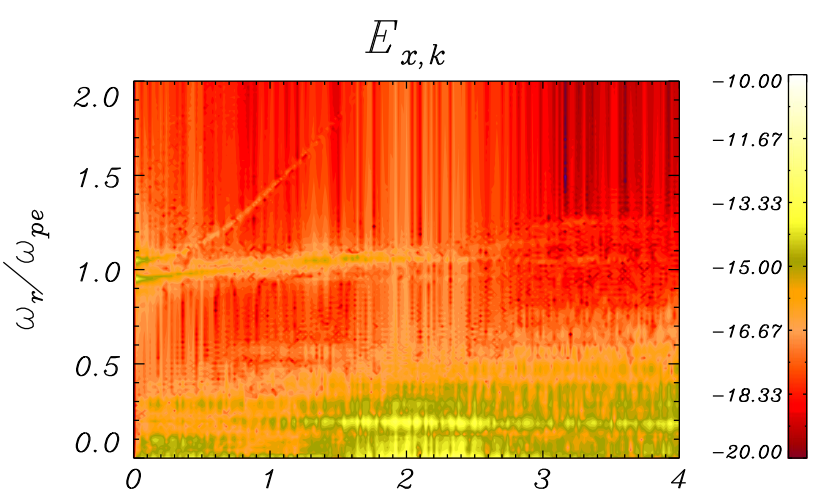

(a) $k c / \omega_{p e}$

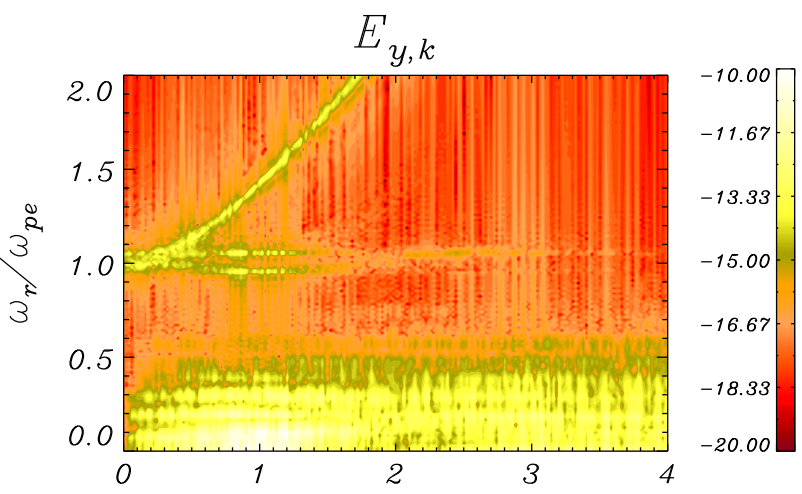

(b) $k c / \omega_{p e}$

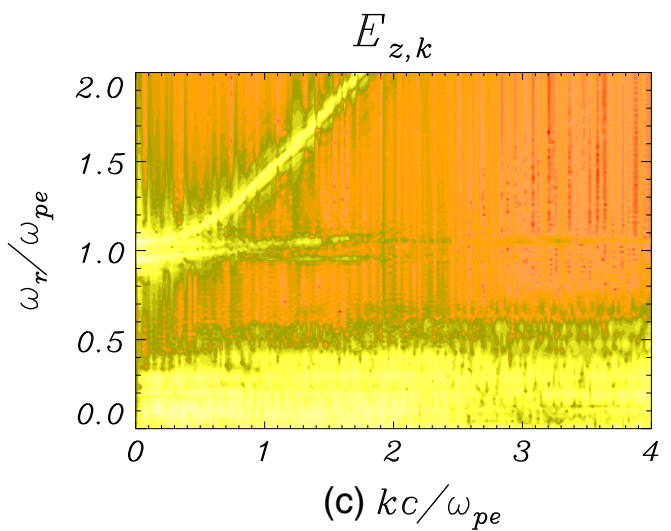

Figure 2. Contour plots of the electric fields in $\left(\omega_{r}-k\right)$ plane for $B_{0}=0.1$.

growth phase of the FI. We observe a clear electromagnetic mode with $\omega \sim k$ propagating along $E_{y, k}$. This is the ordinary mode arising due to the magnetic field present in the system. The FI grows exponentially in time and can nonresonantly drive a wide spectrum of modes, provided their electric field polarisation matches that of the instability. On the other hand, if we see the electric vectors perpendicular to $k$, i.e $E_{z, k}$, the propagation of electromagnetic mode is noticed which is nothing but the $\mathrm{X}$-mode. The propagation of Bernstein mode is clearly noticed in the spectrum of $E_{x, k}$ along with the $\mathrm{X}$-wave. The wave coupling is caused by the presence of multiple wave modes in the magnetised plasma. In a fluid model, we have only the slow extraordinary and the fast extraordinary modes at low wave numbers. The fast X-mode approaches the $\mathrm{O}$-mode at high $k$ while the slow $\mathrm{X}$-mode converges to the upper hybrid frequency at high $k$. In a kinetic framework they encounter the Bernstein mode (electron cyclotron) waves at higher $k$. The Bernstein modes are subdivided in two types. The $n$th extraordinary Bernstein mode starts at the $n$th harmonic of the cyclotron frequency, and its frequency decreases slightly with increase in $k$, eventually converging at high $k$ to the $n$th harmonic of the cyclotron frequency. These modes are electromagnetic in nature. The $n$th ordinary Bernstein modes below the upper hybrid frequency starts at the $(n+1)$ th harmonic of the cyclotron frequency and converges to the $n$th harmonic at large $k$. The $n$th harmonic above the upper hybrid frequency starts at the $n$th harmonic of $\omega_{c e}$, goes through a maximum and converges again with the $n$th harmonic. The ordinary Bernstein modes are electrostatic, except at low $k$ and close to the upper hybrid frequency. The slow X-mode couples to the ordinary Bernstein mode wave branch that contains the upper hybrid frequency. In our result, what we see at low $k<0.1$ is the slow $\mathrm{X}$-mode at $0.95 \omega_{r}$ and the fast $\mathrm{X}$-mode at $1.05 \omega_{r}$. At $k=1$, the two Bernstein mode branches are separated in frequency. To ensure that the coupling is coming from the instability, we have performed a PIC simulation which computes the spectrum for a single-Maxwellian distribution of electrons. Hence, the distribution is stable and not related to any of the instability observed in Vlasov model. What we obtain in the PIC simulation is the noise at a frequency resolution where there is no instability and the growing wave has no effect on it. This noise distribution illustrates the distribution of the Bernstein modes and of the electromagnetic modes as shown in figure 3. No coupling of the waves is observed at high $k$ unlike the Vlasov model. Therefore, unlike the PIC codes, the Vlasov code shows wave-wave coupling which feeds the undamped modes. For further confirmation, we have shown in figure 4 the power spectra for $B=0.2$. If we have no noise, as we assume in the linear dispersion relation, the electron will always orbit around the magnetic field at the electron cyclotron frequency. However, we have noise in the PIC simulation and this affects the particle motion. The particle gets little kicks along the orbit and it changes its velocity in time. The consequence is that the particle does not gyrate with a constant frequency. It gyrates with an average frequency close to the cyclotron frequency. The gyration is no longer characterised by a single frequency but by a distribution of frequencies. Here, 


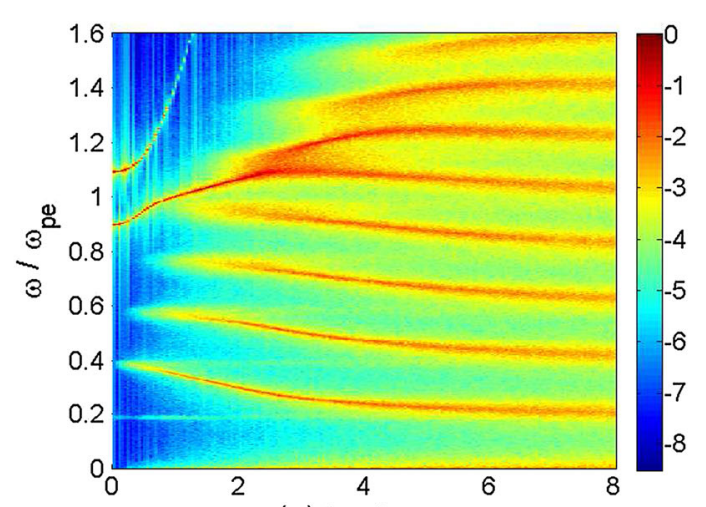

(a) $k \mathrm{c} / \omega_{\text {pe }}$

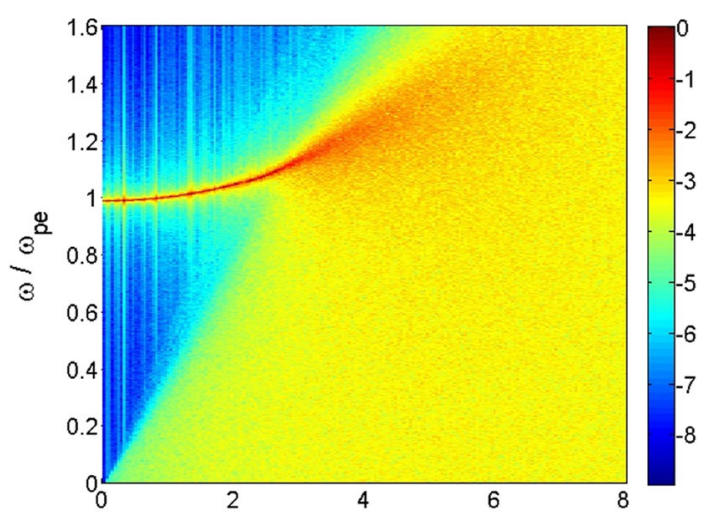

(b) $k \mathrm{c} / \omega_{\mathrm{pe}}$

Figure 3. Results from PIC simulations for the magnetic field spectrum in $\left(\omega_{r}-k\right)$ space. Top panel: $B_{0}=0.2$ and bottom panel: $B_{0}=0.0$.

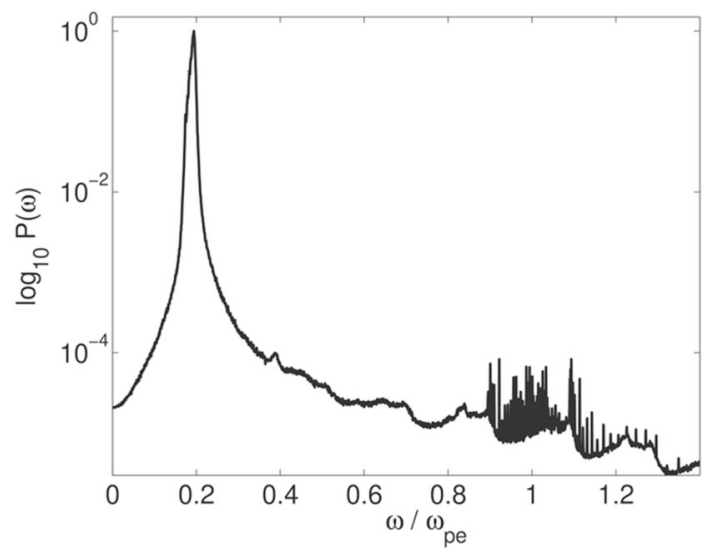

Figure 4. Power spectrum from PIC simulations for the case with $B_{0}=0.2$.

they are perturbed over a broader range due to the impact of the broadband thermal noise. Interestingly, we do not observe this coupling of the filamentation mode to the high-frequency modes for strong magnetic field cases (for example $B_{0}=0.5$ as shown in figure 5). The exponential growth rate of the FI is smaller. Thus,

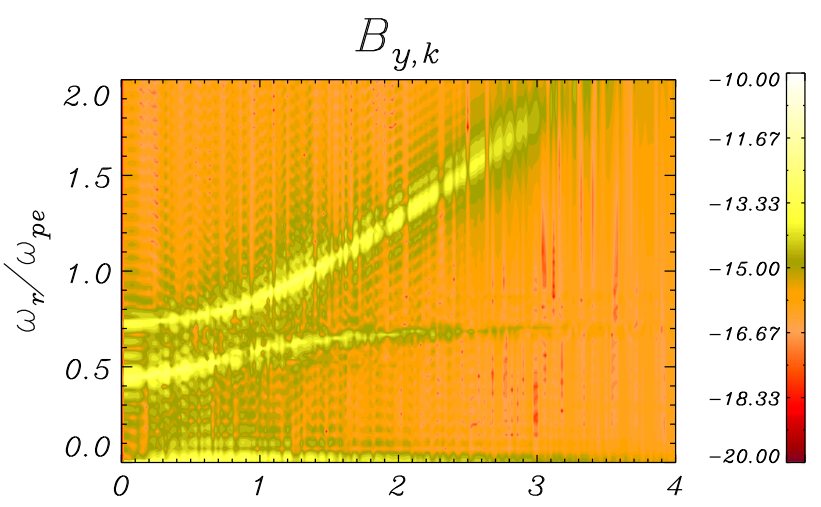

(a) $k c / \omega_{p e}$

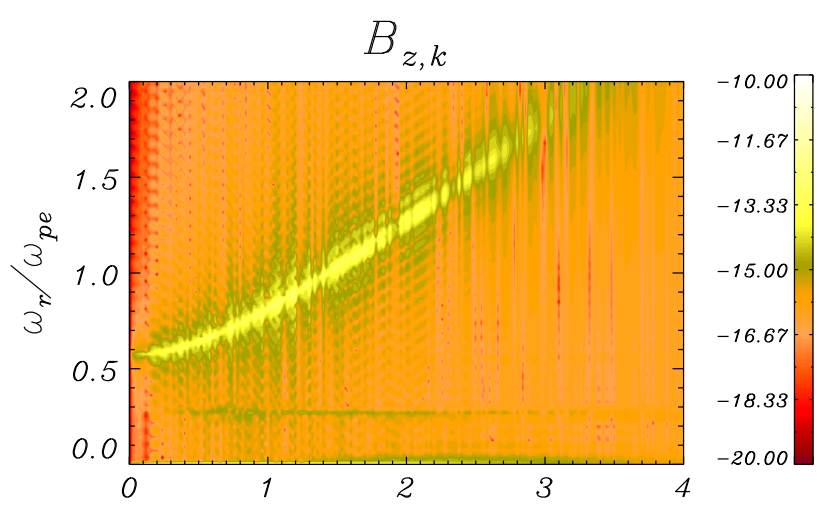

(b) $k c / \omega_{p e}$

Figure 5. Magnetic field spectrum for $B_{0}=0.5$.

the Fourier transform along time gives us a frequency spectrum that is concentrated in lower frequencies. The high-frequency part is much weaker and cannot couple well to the O-mode.

\section{Nonlinear phase of the instability}

The time evolution of Fourier-transformed magnetic fields $B_{z, k}, B_{y, k}$ and electric field $E_{x, k}$ at their corresponding dominant $k$-mode are shown in figure 6 . The perturbed magnetic field $B_{z, k}$ grows exponentially with the growth rate $\gamma_{\max } \sim 0.06$ at the maximum mode $k_{\max }=k_{1}=1$ which start to saturate from $t \sim 150$. At long times the field saturates completely. The scenario for the saturation mechanism can be explained as follows: if the orbits of the particles are straight, the particles can form current filaments, which generate current filaments around themselves. Thus, the magnetic field strength grows consistently as long as the orbits of particles can be regarded as straight. On the other hand, once the magnetic field becomes strong enough to deflect the orbits of the particles, the particles cannot form current filaments. Hence, it is expected that the magnetic field 


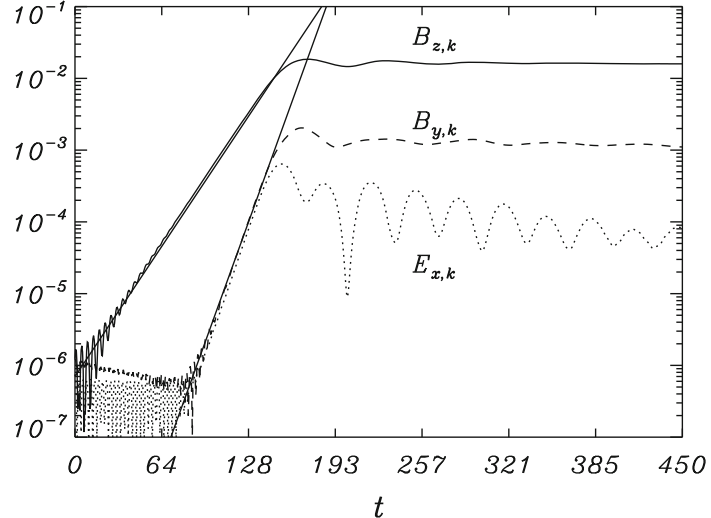

Figure 6. Growth of the magnetic and electric fields for $B_{0}=0.1$.
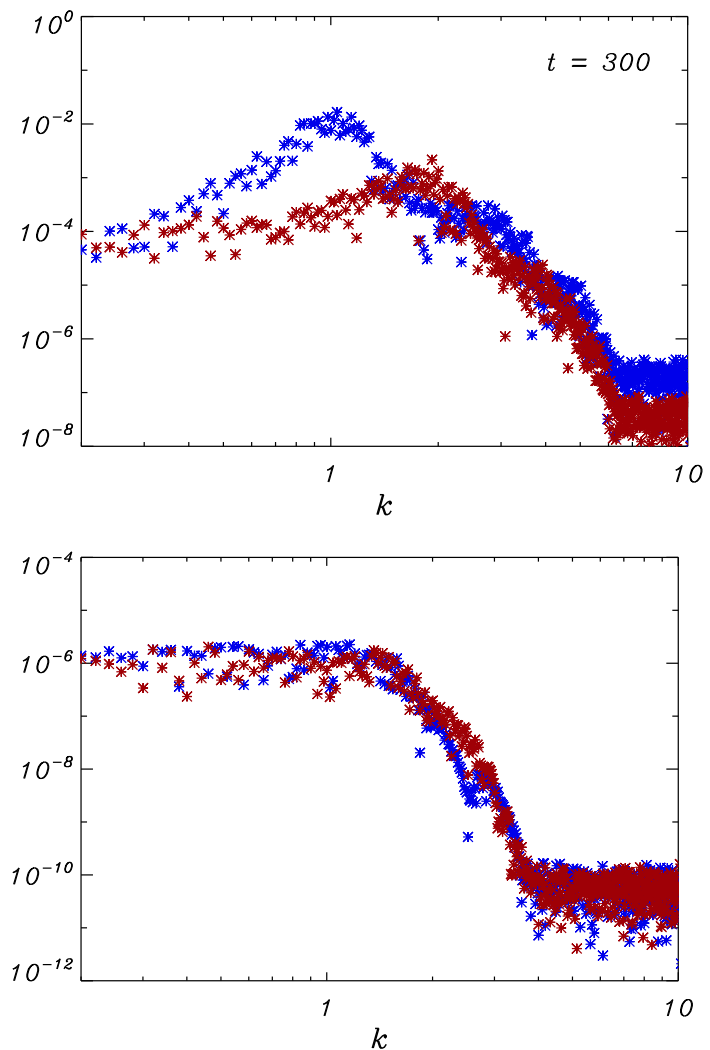

Figure 7. Magnetic field spectrum $B_{z, k}$ and $B_{y, k}$ for $B_{0}=0.1$ (top panel) and $B_{0}=0.5$ (bottom panel). Here, $k$ is normalised to $c / \omega_{\text {pe }}$.

strength will be saturated when the Larmor radius $r_{\mathrm{L}}$ of a particle becomes comparable to the characteristic scale length $l$ of the plasma. An electrostatic field $E_{x, k}$ is also present which does not grow initially and starts growing only after $t \sim 90$ when the magnetic field has reached a large amplitude, at a growth rate which is twice as that of the magnetic field, $\gamma=0.13$ at $k_{2}=k_{\max }=2 k_{1}$. Electron beams flowing in the $y$ direction cannot drive
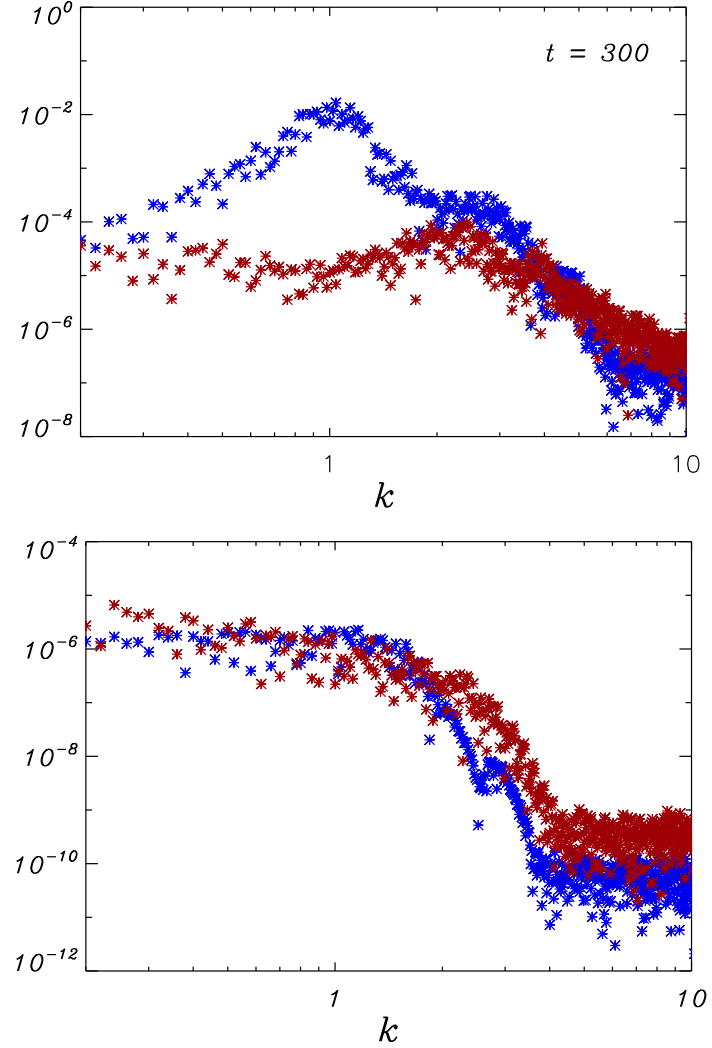

Figure 8. Field spectrum $B_{z, k}$ and $E_{x, k}$ for $B_{0}=0.1$ (top panel) and $B_{0}=0.5$ (bottom panel). Here, $k$ is normalised to $c / \omega_{p e}$.

an electrostatic two-stream instability in the $x$ direction. This implies that $E_{x, k}$ is driven by the FI. After saturation, the electric field starts decreasing accompanied with oscillations. It is interesting to see that the growth of $B_{y, k}$ generating along the streaming beams, has approximately the same growth rate as that of the electrostatic field. The amplitude of $B_{y, k}$ is greater than $E_{x, k}$ but less than $B_{z, k}$. A Fourier spectrum in $k$-space is shown in figure 7 at $t=300$ for $B_{z, k}$ (blue) and $B_{y, k}$ (red) for $B_{0}=0.1$ (top panel) and $B_{z, k}$ (blue) and $B_{y, k}$ (red) for $B_{0}=0.5$ (bottom panel). We see a difference in spectrum for the two different ambient magnetic field cases. For $B_{0}=0.1$, which is lower than the marginal stability magnetic field, $B_{\mathrm{c}}$ has a growth peak at $k \approx 1$ and then stabilises. On the other hand, for $B_{0}=0.5>B_{\mathrm{c}}$, there is no growth of the fields initially, as discussed in the linear regime. However, there is still some stabilisation after $k \approx 1$, which implies that there is some effect of the ambient magnetic field in its saturation region. The same is observed in figure 8 where $B_{z, k}$ (blue) and $E_{x, k}$ (red) for $B_{0}=0.1$ (top panel) and $B_{z, k}$ (blue) and $E_{x, k}$ (red) for $B_{0}=0.5$ (bottom panel) are shown. Unlike the case of $B_{0}=0.1$, when the ambient magnetic field becomes stronger $\left(B_{0}=0.5\right)$, 
at low $k$, the spectrum is dominated by the magnetic field $B_{z, k}$ while in the interval $2 \leq k \leq 5$, the system is dominated by both $E_{x, k}$ and $B_{y, k}$. However, at high $k$, structures become predominantly electrostatic. The $E_{x}$ component grows in a $k$-interval that coincides with the high- $k$ tail of the $B_{z}$ spectrum. The overlap suggests that the $E_{x}$ field is connected to rapid (high$k$ ) variations of the $B_{z}$, while the slow $B_{z}$ variations do not give rise to $E_{x}$. Once $E_{x}$ has reached a high power, both the wave spectra shift towards lower $k$ (not shown).

\section{Conclusions}

This work is mainly focussed on the linear phase of the FI and has shown that at a threshold magnetic field, the FI gets stabilised in Vlasov simulations unlike in PIC simulations where instability continues to grow as the strength of the magnetic field goes on increasing. We considered a 1D-3V system with periodic boundary conditions and involve only electrons in motion in a uniform positively charged background. We found a close agreement of the two methods, i.e. the Vlasov runs and the PIC runs, when the strength of the external magnetic field is low. Both methods show that with increasing ambient magnetic field there is a suppression of the FI. Using Vlasov simulations, we have found the growth rates and the modes at which the growth is maximum for the cases with $B_{0}=0.0,0.1$ and 0.15 . The dispersion relation behaves differently as the system converts from an unmagnetised to magnetised plasma. In the longer wavelength regions it is shown that unlike unmagnetised plasma, in the magnetised plasma, stabilisation of instability is stronger when the thermal velocity is on the higher side than when the thermal velocity is lower.

Most interestingly, at a threshold/critical magnetic field: $\omega_{c e}=\left(\omega_{p e} / c\right)\left(v_{0}^{2}+v_{\text {the }}^{2} / 2\right)^{1 / 2}$ it is seen that the PIC simulations still show a growth in the instability. However, Vlasov simulation shows a complete rescindment of the same. Considering the fact that PIC simulations have thermal noise in them, which may be the reason for the instability generation, some initial random noise is added continuously to the Vlasov code at regular intervals. But, the simulations do not show any kind of instability development at the critical magnetic field, suggesting a different mechanism for the growth of instability in the PIC simulation. This argument is verified with a detailed spectral analysis using Vlasov simulations and comparing with the PIC results. The thermal noise in the PIC simulations is due to the granular structure of the phase-space density distribution and reflects the random motion of individual particles. On the other hand, Vlasov model has random waves in the system, which propagate as Bernstein waves and upper hybrid waves. These waves couple with each other and this wave-wave coupling leads to the stabilisation of the system. However, at a very strong ambient magnetic field, because of the weak FI, high frequency range is unsubstantial to couple with the ordinary mode. Some initial results on the effect of background magnetic field on the stabilisation of instability is also shown in the saturation phase. However, a detailed explanation and analyses of the nonlinear regime of the FI is in progress and will be presented in our next work.

\section{References}

[1] E S Weibel, Phys. Rev. Lett. 2, 83 (1958)

[2] M V Medvedev, L O Silva and M Kamionkowaski, Astrophys. J. 642, L1 (2006)

[3] M V Medvedev and A Loeb, Astrophys. J. 526, 697 (1999)

[4] M V Medvedev, Astrophys. Space Sci. 307, 245 (2007)

[5] J Arons, AIPC 983, 200 (2008)

[6] G A Askar'yan, S V Bulanov, F Pegoraro and A M Pukhov, JETP Lett. 60, 240 (1994)

[7] G A Askar'yan, S V Bulanov, F Pegoraro and A M Pukhov, Commun. Plasma Phys. Contr. Fus. 17, 35 (1995)

[8] F Califano, N Attico, F Pegoraro, G Bertin and S V Bulanov, Phys. Rev. Lett. 86, 5293 (2001)

[9] S Belghit and A Sid, Pramana - J. Phys. 87: 96 (2016)

[10] N Ahmad, V K Tripathy, Moiz Ahmad and M Rafat, Pramana - J. Phys. 86, 157 (2016)

[11] M Strickland, Pramana - J. Phys. 84, 671 (2015)

[12] S Saito and J I Sakai, Phys. Plasmas 11, 5547 (2004)

[13] F Califano, F Pegoraro and S V Bulanov, Phys. Rev. E 56, 963 (1997)

[14] F Califano, F Pegoraro, S V Bulanov and A Manganey, Phys. Rev. E 57, 7048 (1998)

[15] R C Davidson and D A Hammer, Phys. Fluids 15, 317 (1972)

[16] F Califano, T Cecchi and C Chiuderi, Phys. Plasmas 9 , 451 (2002)

[17] G Rowlands, M E Dieckmann and P K Shukla, New J. Phys. 9, 247 (2007)

[18] M E Dieckmann, G Rowlands, I Kourakis and M Borghesi, Phys. Plasmas 16, 074502 (2009)

[19] M Honda, J Meyer-ter-Vehn and A Pukhov, Phys. Plas$\operatorname{mas} \mathbf{7}, 1302$ (2000)

[20] J I Sakai, R Schlickeiser and P K Shukla, Phys. Lett. A 330, 384 (2004)

[21] M Murakami and L M Lidsky, Phys. Rev. Lett. 24, 7 (1970)

[22] A Bret, Phys. Plasmas 14, 032103 (2007)

[23] L Palodhi, F Califano and F Pegoraro, Plasma Phys. Control. Fusion 51, 125006 (2009)

[24] A Stockem, M E Dieckmann and R Schlickeiser, Plasma Phys. Control Fusion 50, 025002 (2008) 
[25] S Ichimaru, Ann. Phys. 20, 78 (1962)

[26] B Coppi and F Pegoraro, Ann. Phys. 134, 376 (1981)

[27] W M Nevins, G W Hammett, A M Dimits, W Dorland and D E Shumaker, Phys. Plasmas 12, 122305 (2005)
[28] A Bottino, A G Peeters, R Hatzy, S Jolliet, B F McMillan, T M Tran and L Villard, Phys. Plasmas 14, 010701 (2007)

[29] T H Dupree, Phys. Fluids 6, 1714 (1963) 